\title{
Inventario herpetológico en hábitats mediterráneos de un municipio del sur de Álava (País Vasco).
}

\section{Herpetological inventory in the Mediterranean habitats of a municipality in southern Alava (Basque Country).}

Gabriel García de Marcos ${ }^{1}$, Ion Garin-Barrio², Ernesto Pérez Collazos ${ }^{3}$

$\xi^{*}$

\section{Resumen}

La todavía frecuente escasez de datos de biodiversidad en los territorios municipales ha propiciado la realización de un inventario herpetológico en los cuatro tipos de hábitat más significativos del término de Navaridas (Álava) (laguna, carrascal, matorral mediterráneo y viñedo). Entre 2015 y 2016 se llevaron a cabo dos tipos de muestreo (transectos y mangueos de puntos de agua) para identificar las especies, que permitieron la obtención de estimaciones poblacionales. Se encontraron 15 especies, ocho de anfibios y siete de reptiles (39 \% de los herpetos del País Vasco). Los dos espacios protegidos con los que cuenta el municipio (laguna y carrascal de Navaridas) presentaron una mayor diversidad biológica, destacando especialmente la laguna. Los resultados sugieren un posible beneficio de los linderos con muros bajos de piedra entre los viñedos para la especie Podarcis liolepis, que mostró las máximas abundancias (71,5 individuos / ha). El presente estudio aporta una base de conocimiento para el desarrollo de futuros proyectos de inventariado basados en

\footnotetext{
1 c/ Chile, 12, 2 A izda. 26005. Logroño

*Correspondencia: gabrigdm@hotmail.com

2 Departamento de Herpetología. Sociedad de Ciencias Aranzadi c/ Zorroagagaina, 11. 20014. Donostia-San Sebastián *Correspondencia: igarin@aranzadi.eus

3 Departamento de Ciencias Agrarias y del Medio Natural. Escuela Politécnica de Huesca. Universidad de Zaragoza *Correspondencia: ernextop@unizar.es
} 
densidades poblacionales de herpetos, su evolución y la evaluación de la incidencia que la actividad agrícola podría tener sobre dichas poblaciones.

Palabras clave: anfibios, conservación, diversidad, humedal, Podarcis liolepis, reptiles, Rioja Alavesa, viñedos.

\begin{abstract}
The frequent lack of biodiversity data on municipal areas favoured the performance of a herpetological study to determine the number of herpetological species in the four most significant types of habitat within the municipality of Navaridas (province of Alava). A number of different sampling surveys were performed (transects, and water net points) during 2015 and 2016. 15 herpetological species (8 amphibians and 7 reptiles) were found. Protected areas showed higher diversity levels, especially in the lake and associated holm oak woodland of Navaridas. The results suggest the possible benefit of low stone walls between vineyard for Podarcis liolepis. This study establishes a starting point for further research projects based on the population densities and evolution of herpetological species, and an assessment of the possible effects of agricultural activities on these populations.
\end{abstract}

Key words: amphibians, conservation, diversity, wetland, Podarcis liolepis, reptiles, Rioja Alavesa, vineyards.

\title{
Laburpena
}

Udalerrien mailan biodibertsitate datuak oraindik ere sarritan eskasak izaten direnez, horrek inbentario herpetologiko bat egitea ekarri du, Arabako Navaridas udalerriko lau habitat motatan (aintzira, artadi, sastrakadi mediterraniarra eta mahastiak). 2015-2016 bitartean bi laginketa burutu ziren (transektoak, ur puntuetako mangeoak) espezieak identifikatzeko, eta horien bidez jakin ziren populazio kopuruak. 15 espezie aurkitu ziren, zortzi anfibioenak eta zazpi narrastienak (EHko herpetoen \%39). Udalerriak dituen bi eremu babestuetan (aintzira eta Navaridaseko karraskadia) dibertsitate biologiko handiagoa dago, batez ere aintziran. Emaitzen arabera, badirudi mahastien arteko bidexken ertzetako harrizko horma baxuak onuragarriak direla espezie honentzat: Podarcis liolepis Boulenger, 1905; horrek erakutsi zuen kopuru handiena (71,5 ale/ha). Ikerketa hau ezagutza aldetik oinarri sendo bat da etorkizunera begira inbentario proiektu berriak gauzatzeko, besteak beste espezie desberdinen populazio dentsitateei buruzkoak, horien eboluzioaz eta nekazaritza ekimenek populazio horiengan ze eragin duten neurtzeko.

Gako hitzak: anfibioak, kontserbazioa, dibertsitatea, hezegunea, Podarcis liolepis, narrastiak, Arabar-Errioxa, mahastiak. 


\section{Introducción}

El limitado conocimiento de la diversidad biológica es una de las principales causas que ha dificultado los avances en materia de conservación, manejo y uso sustentable de la biodiversidad a nivel mundial (Conabio, 2000). En este sentido, es importante incrementar los conocimientos básicos sobre los ecosistemas, sus especies y poblaciones, por lo que resulta imprescindible incidir en las labores de inventariado, organización de colecciones científicas y seguimiento sistemático de las poblaciones, como fuentes principales para la documentación de nuestro conocimiento sobre la diversidad biológica.

En el País Vasco la iniciativa municipal para el desarrollo de inventarios herpetológicos en sus territorios es un fenómeno que acaba de iniciarse, del que todavía se cuenta con escasas aportaciones (Garin-Barrio et al., 2010, 2017; Gosá \& Garin-Barrio, 2016). En Navaridas apenas se cuenta con estudios relacionados con su patrimonio natural, incluidos los de sus espacios protegidos (García de Marcos et al., 2016), por lo que el presente estudio contribuiría en la gestión y uso adecuado de dichas áreas. El principal objetivo planteado es la realización de un inventario herpetológico en los cuatro hábitats representativos del municipio de Navaridas (laguna, carrascal, matorral mediterráneo y viñedo), determinando la riqueza específica y estimando ciertos parámetros relacionados con su ecología, como la diversidad y equitatividad en cada uno de ellos.

\section{Área de estudio y métodos}

El área de estudio se encuentra en el municipio de Navaridas (Álava, País Vasco), abarcando cuatro tipos de hábitats: viñedo, matorral mediterráneo, carrascal y un humedal (Laguna de Navaridas), situados dentro de las cuadrículas UTM (10 x 10 km) WN20 y WN21. Dos de estos cuatro hábitats se corresponden con las zonas protegidas del municipio (Laguna y Carrascal de Navaridas; Fig. 1).

Se realizaron idénticos transectos mensuales de anfibios y reptiles en los cuatro hábitats estudiados por un mismo recorrido de forma sistemática, en otoño de 2015 (octubre, noviembre) y primavera de 2016 (marzo, abril, mayo), por un único observador, en horas diurnas y bajo condiciones meteorológicas apropiadas para la observación de la herpetofauna, dependiendo de las fechas. Los transectos se repitieron un mínimo de dos veces por mes, su longitud aproximada fue de 900 m y su duración de $1 \mathrm{~h}$. Los resultados se han expresado en densidad máxima relativa (número de individuos adultos / ha), a partir de una banda de $2 \mathrm{~m}$ a ambos lados del observador. El transecto de la Laguna transcurrió entre carrizales y zonas de viñedo; el del Carrascal entre carrascal mediterráneo seco y roquedos; el del matorral mediterráneo entre romerales, aliagares y lastonares de Brachypodium sp. con presencia de muros de piedra; y el del viñedo por lindes entre viñedos con presencia de herbazales de Brachypodium sp. y muros bajos de piedra. Los transectos se repitieron en 14 ocasiones en el humedal, el 


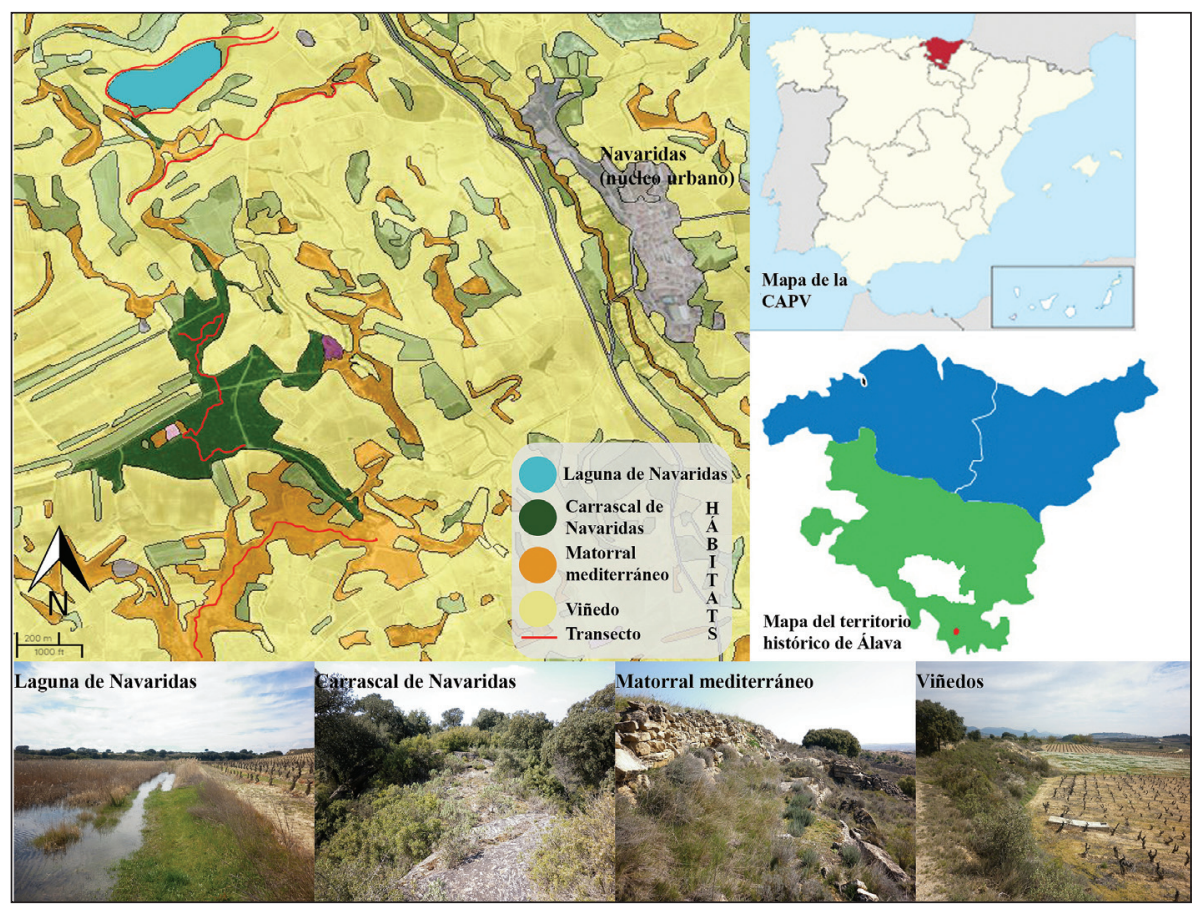

Fig. 1.- Localización geográfica del municipio de Navaridas (derecha) y ubicación del área de estudio con los cuatro hábitats estudiados (izquierda).

Fig. 1.- Geographical location of the municipality of Navaridas (right) and the location of the study area with the four habitats studied (left).

matorral mediterráneo y el viñedo, y en 16 en el carrascal. Los anfibios se muestrearon, además, mediante mangueos con salabre en cuatro áreas diferenciadas de la laguna de Navaridas, entre agosto y octubre de 2015 y mayo-junio de 2016: (1) orilla (profundidad de $10 \mathrm{~cm}$ ), (2) puntos con vegetación lacustre (profundidad de $50 \mathrm{~cm}$ ), (3) zona interior con lámina de agua (profundidad de $60 \mathrm{~cm}$ ) y (4) el canal de desagüe (profundidad de $20 \mathrm{~cm}$ ). Los resultados se han expresado como abundancias máximas (número de individuos / 15 minutos de muestreo), separadas para adultos y larvas. Se realizaron en cada una de las áreas de forma sistemática dos mangueos diurnos y otros dos nocturnos por mes, cada uno con una duración de 15 minutos. De forma complementaria a los transectos y mangueos, se realizaron escuchas mensuales de coros en la Laguna de Navaridas, que permitieron la detección del inicio de la actividad reproductora de algunas especies de anuros, aportando información para el establecimiento del calendario de censos.

Una vez extraídos los datos de los transectos se determinó la diversidad y equitatividad conjunta para anfibios y reptiles en cada hábitat, mediante la aplicación respectiva de los índices de Shannon-Wiener y J de Pielou. 


\section{Resultados}

\section{Inventario herpetológico y densidades poblacionales}

Se detectaron un total de 15 especies de herpetos, ocho de ellas anfibios (Triturus marmoratus (Latreille, 1800), Lissotriton helveticus (Razoumowsky, 1789), Alytes obstetricans (Laurenti, 1768), Pelodytes punctatus (Daudin, 1802), Bufo spinosus (Daudin, 1803), Epidalea calamita (Laurenti, 1768), Pelophylax perezi (López Seoane, 1885) e Hyla molleri (Bedriaga, 1889)), y siete reptiles (Chalcides striatus (Cuvier, 1829), Podarcis liolepis (Boulenger, 1905), Psammodromus algirus (Linnaeus, 1758), Timon lepidus (Daudin, 1802), Rhinechis scalaris (Schinz, 1822), Malpolon monspessulanus (Hermann, 1804) y Natrix astreptophora (López Seoane, 1884)). El hábitat con mayor riqueza herpetológica fue la Laguna de Navaridas, con diez especies.

Se detectaron un total de 524 individuos mediante la metodología de transectos. El número de observaciones obtenidas fue netamente superior para los reptiles $(77,1$ $\% ; n=404)$ en comparación a los anfibios $(22,9 \% ; n=120)$ en el conjunto de los hábitats.

Como era de esperar, el mayor número de individuos de anfibios se detectó en la Laguna de Navaridas, albergando el 86,7 \% del total de las observaciones para este grupo ( $n=104$; Fig. 2). En el caso de los reptiles el hábitat que acogió el mayor número de avistamientos fue el matorral mediterráneo, con el 40,3 \%, siendo el más pobre la Laguna de Navaridas (Fig. 2).

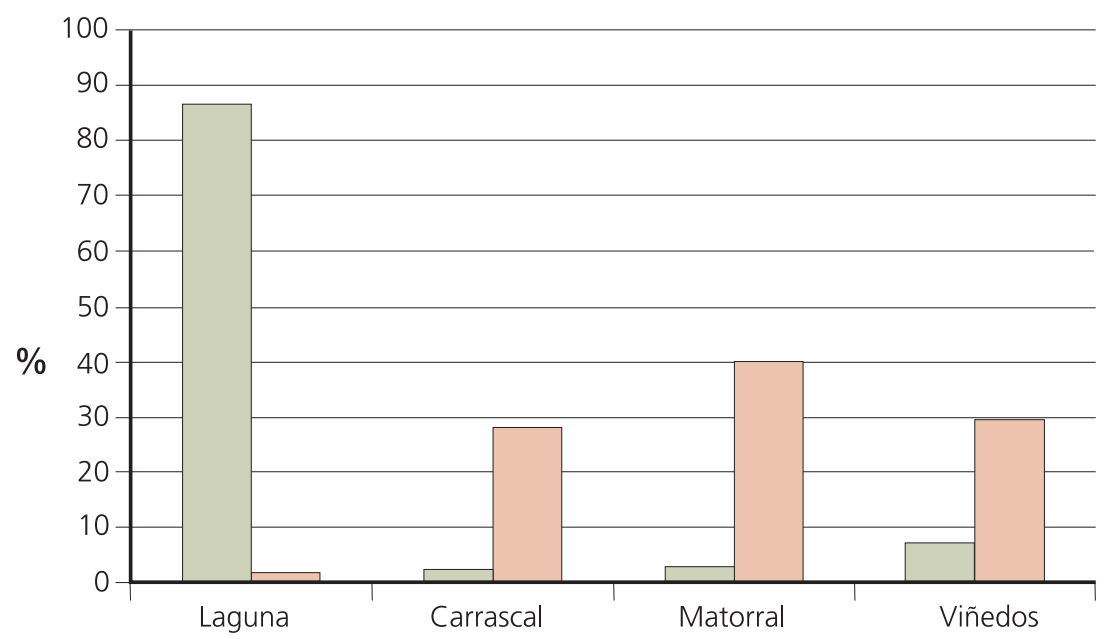

Fig. 2.- Uso del hábitat (\%) por los anfibios (verde) y reptiles (naranja), obtenido a partir de transectos.

Fig. 2.- Habitat use (\%) by amphibians (green) and reptiles (orange), obtained from transect data. 
Pelophylax perezi fue la especie de anfibio de la que se obtuvo el mayor porcentaje de observaciones para el conjunto de los cuatro hábitats, con un 75,8 \% de los avistamientos ( $n=91)$. Entre los reptiles la especie mejor representada fue Podarcis liolepis, con un 91,1 \% de observaciones ( $n=368$; Fig. 2).

La densidad relativa máxima de anfibios en los transectos solo superó los 10 individuos / ha en la laguna, siendo $P$. perezi la especie más abundante. Por el contrario, y como era de esperar, la densidad máxima más baja en reptiles se registró en este hábitat acuático, donde el conjunto de observaciones no superó los 10 ind. / ha, mientras que en los restantes hábitats se registraron más de 60 ind. / ha. Prácticamente ninguna especie de reptil superó los 10 ind. / ha, salvo $P$. liolepis que, excepto en la laguna, estuvo por encima de 39 ind. / ha (Tabla 1). Mediante la técnica de mangueos se detectaron seis de las ocho especies presentes de anfibios, correspondiendo el 51,1 $\%$ de las capturas a individuos en fase aérea (adultos y juveniles). Pelophylax perezi

\begin{tabular}{|lcccc|}
\hline Transectos: anfibios & Laguna & Carrascal & Matorral & Viñedo \\
\hline Pelophylax perezi & 43,5 & 0,0 & 0,0 & 0,0 \\
Alytes obstetricans & 2,7 & 2,6 & 0,0 & 0,0 \\
Epidalea calamita & 2,7 & 2,6 & 2,7 & 2,6 \\
Pelodytes punctatus & 2,7 & 0,0 & 2,7 & 0,0 \\
Bufo spinosus & 16,3 & 2,6 & 0,0 & 0,0 \\
Triturus marmoratus & 0,0 & 0,0 & 0,0 & 2,6 \\
Lissotriton helveticus & 0,0 & 0,0 & 0,0 & 2,6 \\
Total & 67,9 & 7,8 & 5,4 & 7,8 \\
\hline Transectos: reptiles & Laguna & Carrascal & Matorral & Viñedo \\
\hline Podarcis liolepis & 5,4 & 39,5 & 57,3 & 71,5 \\
Psammodromus algirus & 0,0 & 7,9 & 8,2 & 5,3 \\
Timon lepidus & 0,0 & 0,0 & 0,0 & 2,6 \\
Chalcides striatus & 0,0 & 5,3 & 8,2 & 0,0 \\
Rhinechis scalaris & 0,0 & 0,0 & 2,7 & 0,0 \\
Malpolon monspessulanus & 0,0 & 10,5 & 2,7 & 0,0 \\
Natrix astreptophora & 2,7 & 0,0 & 0,0 & 0,0 \\
Total & 8,1 & 63,2 & 79,1 & 79,4 \\
\hline
\end{tabular}

Tabla 1- Densidad relativa máxima de anfibios y reptiles ( $n^{\circ}$ individuos / ha), obtenida en los transectos y distribuida por hábitats. Número de transectos por hábitat: laguna de Navaridas, $n=14$; carrascal, $\mathrm{n}=16$; matorral mediterráneo, $\mathrm{n}=14$; viñedos, $\mathrm{n}=14$.

Table 1- Maximum relative density of amphibians and reptiles (ind/ha), obtained by transects and by habitat. Number of transects by habitat: Navaridas lake, $n=14$; holm oak forest, $n=16$; Mediterranean scrub, $n=14$; vineyard $=14$. 
fue la especie con mayor número de registros (23,5 \% en fase aérea y 76,5 \% en fase larvaria; $n=17$ ) junto a $T$. marmoratus (93,8 \% en fase aérea y $6,2 \%$ en fase larvaria; $\mathrm{n}=16$; Tabla 2). Las escuchas de coros nocturnos permitieron detectar la presencia de Hyla molleri en la Laguna de Navaridas.

\begin{tabular}{|lcc|}
\hline Especies & Adultos & Larvas \\
\hline Pelophylax perezi & 4 & 13 \\
\hline Alytes obstetricans & 0 & 3 \\
\hline Pelodytes punctatus & 2 & 1 \\
\hline Epidalea calamita & 1 & 0 \\
\hline Triturus marmoratus & 15 & 1 \\
\hline Lissotriton helveticus & 2 & 5 \\
\hline
\end{tabular}

Tabla 2.- Abundancia máxima de anfibios ( $n^{\circ}$ ind. / 15 minutos), obtenida por mangueo con salabre en la laguna de Navaridas. Número de mangueos por área: orilla, $n=20$; puntos con vegetación lacustre, $n=20$; zona interior con lámina de agua, $n=20$; canal de desagüe, $n=4$.

Table 2.- Maximum abundance of amphibians (ind/15 min.), obtained by net sampling in Navaridas lake. Number of water net passes by area: shore, $n=20$; lacustrine vegetation points, $n=20$; indoor area with water, $n=20$; drainage canal, $n=4$.

\section{Diversidad y equitatividad}

La diversidad y equitatividad para el conjunto de especies muestran valores máximos en los dos espacios protegidos, la Laguna y el Carrascal, frente al menor en los viñedos (Tabla 3).

\begin{tabular}{|lcccc|}
\hline & Laguna & Carrascal & Viñedos & $\begin{array}{c}\text { Matorral } \\
\text { mediterráneo }\end{array}$ \\
\hline Diversidad & 1.90 & 1.90 & 1.09 & 1.67 \\
Equitatividad & 0.68 & 0.68 & 0.42 & 0.60 \\
\hline
\end{tabular}

Tabla 3.- Diversidad y equitatividad herpetológica en los cuatro hábitats estudiados del municipio de Navaridas.

Table 3.- Herpetological diversity and evenness in the four habitats studied in the municipality of Navaridas.

\section{Discusión}

\section{Inventario herpetológico}

El municipio de Navaridas cuenta con 8 de las 9 especies de anfibios de la Rioja Alavesa, lo que representa casi la mitad de las 17 existentes en la Comunidad Autónoma del País Vasco. Esta circunstancia refleja la importancia que estas zonas tienen en el contexto regional. 
Los muestreos realizados no han detectado la presencia de dos especies de anfibios históricamente conocidas en la Rioja Alavesa, que podrían encontrarse en la zona de estudio: el sapo de espuelas Pelobates cultripes (Cuvier, 1829) y el sapillo pintojo Discoglossus galganoi (Capula, Nascetti, Lanza, Bullini y Crespo, 1985). Las poblaciones del Alto Ebro de ambas especies se sitúan en su límite septentrional de distribución ibérica, presentando graves problemas para su subsistencia. Dos de sus principales amenazas pueden tener origen biogeográfico: la elevada fragmentación de sus poblaciones y el bajo número de ejemplares de sus núcleos reproductores (Crespo, 2008). Pero si algo caracteriza al Alto Ebro (y por tanto a la Rioja Alavesa) es la pérdida y alteración del hábitat, principalmente de los medios acuáticos, que si no han sido destruidos por desecación (sobre todo los pequeños encharcamientos que se forman en el borde de los cultivos), sufren procesos periódicos de contaminación derivada de los productos agroquímicos utilizados en los cultivos monoespecíficos (Crespo et al., 2007; Crespo, 2008). En el caso de Pelobates cultripes, la última cita de la que se tiene constancia en la Rioja Alavesa data del año 2003 (EKOS, 2005; Olano et al., 2007). No obstante, se ha podido confirmar la presencia de Pelobates cultripes en la cercana laguna de Lacorzana (término de Armiñón, Álava), enclave de similares características a las de Navaridas (I. Garin, no publ.), por lo que no es descartable la presencia de una población exigua de esta especie en el territorio estudiado.

La situación de Discoglossus galganoi es crítica en la CAPV, donde tiene pendiente la actualización de su catalogación, así como en los correspondientes listados autonómicos de La Rioja y Navarra. Es, sin duda, uno de los anfibios más amenazados en el Alto Ebro (Garin-Barrio et al., 2013). En La Rioja y Navarra no se ha vuelto a observar en el siglo XXI, y las citas para la CAPV se circunscriben a enclaves puntuales del sur de Álava, fuera de la Rioja Alavesa (Garin-Barrio et al., 2013). Durante el quinquenio 2007-2012 la Sociedad de Ciencias Aranzadi ha realizado un trabajo de seguimiento en la zona alta del Valle del Ebro, incluida la Laguna de Navaridas, por lo que sería de interés continuar prospectando este humedal de forma periódica.

Por otra parte, se ha constatado en Navaridas la presencia de 7 de las 21 especies de reptiles de la CAPV y la mitad de las 14 reportadas para la Rioja Alavesa, por lo que la conservación y buena gestión de estos hábitats también resultaría relevante y prioritaria. Además, se ha añadido una cita de Natrix astreptophora para una nueva cuadrícula en el País Vasco (WN21) (SIARE, 2018). El comportamiento esquivo de los reptiles, su escasa movilidad diaria y su habilidad para pasar inadvertidos han dificultado su muestreo. A pesar de que se conoce la presencia de Anguis fragilis (Linnaeus, 1758) en la zona de estudio (Bea, 1985), nuestros muestreos no lo han registrado, como ha ocurrido con Vipera aspis (Linnaeus, 1758), Coronella girondica (Daudin, 1803) y Natrix maura (Linnaeus, 1758), también citadas, aunque estas dos últimas especies se han encontrado en otras zonas del municipio en el mismo periodo de estudio (García de Marcos et al., 2016). 


\section{Diversidad y equitatividad}

Los índices de diversidad, en particular el relativo, son de tipo medio en espacios protegidos (la Laguna y el Carrascal de Navaridas), y bajo en las zonas más alteradas por el hombre (viñedos; Tabla 3). Los valores intermedios obtenidos para el matorral mediterráneo serían congruentes con los de un hábitat alterado por la actividad antrópica años atrás, y que por abandono podría estar recuperando las características ecológicas que presentan las áreas naturalizadas, donde se genera una sucesión de las comunidades vegetales y faunísticas autóctonas del entorno. Estas diferencias de diversidad entre hábitats naturalizados y alterados, enfocadas desde un punto de vista ecológico, afianzan la idea ya propuesta por diversos autores (De la Montaña \& Rey-Benayas, 2002; Lambert, 2003; Kati et al., 2007; Carvajal-Cogollo \& Urbina-Cardona, 2008), por la que los espacios naturales, humedales y remanentes de lo que un día fue el tipo de bosque autóctono, coinciden generalmente con las zonas de mayor diversidad herpetofaunística. Sin embargo, estos resultados deben tomarse con prevención y no deben ser considerados como representativos de una región, ya que los datos han sido recogidos en un área reducida de la Rioja Alavesa, y siendo tan escasos en algunos de los hábitats no aportarían una estimación contrastada de la situación de las poblaciones de herpetos en ellos. No obstante, nuestros muestreos han establecido indicios de una tendencia decreciente en cuanto a la riqueza específica, a medida que el medio se encuentra más antropizado, en sentido carrascal-matorral mediterráneo-viñedo. Otros estudios también han incidido en que los valores bajos de equitatividad indicarían una baja representación específica y caracterizarían hábitats que han sufrido recientemente un proceso de colonización y/o alteración (Pineda et al., 2005; ViteSilva et al., 2010). El reducido valor encontrado en el viñedo coincidiría con las diferencias obtenidas para otras especies de anfibios entre hábitats naturales y hábitats en los que se han implantado modelos agrícolas modernos (Pineda et al., 2005).

\section{Densidad poblacional de Podarcis liolepis}

Podarcis liolepis fue registrada como la especie más abundante, presentando su mayor densidad en los viñedos (Tabla 1). El conocido carácter antrópico de la especie sería corroborado por este dato, por lo que se puede interpretar que este hábitat alterado beneficiaría a la especie. Dado que los propios cultivos en sí son un medio muy pobre para los reptiles, al igual que para sus presas (Crespo-Diaz, 2009), ambos confluyen en un espacio reducido a los linderos entre viñedos, lo que podría facilitar la alimentación de los primeros. Podarcis liolepis prefiere lugares despejados con poca vegetación arbustiva y sustratos de areniscas (Gosá \& Bergerandi, 1994), los cuales están bien representados en esta zona en los linderos de los viñedos, provistos de pequeños muros, paredes rocosas y con escasa o nula vegetación arbórea. La capacidad de acogida en los viñedos en explotación, para los reptiles en general, se concentra en los 
microhábitats de sus lindes, en especial en los muros de piedra arenisca (Crespo-Diaz, 2009), los cuales aportan también refugio, y en los que se ha podido contabilizar el $79 \%$ de los individuos de $P$. liolepis.

\section{Agradecimientos}

A la Sociedad de Ciencias Aranzadi y a Alberto Gosá por la revisión del presente artículo.

\section{Bibliografía}

Bea, A. 1985. Atlas de los Anfibios y Reptiles de Álava, Vizcaya y Guipúzcoa. En: Atlas de los Vertebrados Continentales de Álava, Vizcaya y Guipúzcoa. Álvarez, J., Bea, A., Faus, J.M., Castién, E., Mendiola, I. (Ed.): 55-100. Gobierno Vasco. Vitoria-Gasteiz.

Carvajal-Cogollo, J.E., Urbina-Cardona, J.N. 2008. Patrones de diversidad y composición de reptiles en fragmentos de bosque seco tropical en Córdoba, Colombia. Tropical Conserv. Sci. 1(4): 397-416.

Conabio. 2000. Estrategia nacional sobre biodiversidad de México. Comisión Nacional para el Conocimiento y Uso de la Biodiversidad. México.

Crespo, A. 2008. Identificación de poblaciones, distribución y estado de conservación del sapo de espuelas (Pelobates cultripes) y los sapillos pintojos (Discoglossus spp.) en Álava. Diputación Foral de Araba. Informe inédito.

Crespo, A., González, S., Iraola, A. 2007. Identificación de poblaciones, distribución y estado de conservación de los sapillos pintojos (Discoglossus sp.) en el País Vasco. Gobierno Vasco. Informe inédito.

Crespo-Diaz, A. 2009. Presencia y estado de las poblaciones de reptiles mediterráneos en la Rioja Alavesa. Ihobe. Informe inédito.

De la Montaña, E., Rey-Benayas, J.M. 2002. ¿Coinciden los espacios naturales protegidos con las áreas relevantes de diversidad de herpetofauna en España peninsular y Baleares? Revista Ecosistemas 11(2). Disponible en:

https://rua.ua.es/dspace/bitstream/10045/9812/1/ECO_11(2)_07.pdf

EKOS Estudios Ambientales, S.L. 2005. Censo y caracterización de las poblaciones de tritón pirenaico y sapo de espuelas en la Comunidad Autónoma Vasca. Gobierno Vasco. Informe inédito.

García de Marcos, G., Pérez Collazos, E., Garin-Barrio, I. 2016. Inventario herpetológico de dos zonas protegidas y su entorno en el municipio de Navaridas (Álava). Trabajo de Fin de Grado en Ciencias Ambientales. Universidad de Zaragoza. 
Garín-Barrio, I., Rubio, X., Sanz-Azkue, I., Crespo-Diaz, A., Gosá, A. 2010. Herpetofauna. En: Azpiroz, M. (coord.). Análisis preliminar de la diversidad biológica en el entorno natural de Donostia-San Sebastián. 2008-2009. Vol. 1: Resultados: 194-273. Ayuntamiento de Donostia-San Sebastián.

Garin-Barrio, I., Zaldívar, C., Zaldívar, R., Zuazo, O., Remírez, J.A., Gosá, A., Crespo-Diaz, A., Tejado, C., Potes, M.E. 2013. El declive del sapillo pintojo meridional en el Alto Ebro. Quercus, 332: 26-33.

Garin-Barrio, I., Gosá, A., Rubio, X., Cabido, C. 2017. Errenteriko udalerriko inbentario herpetologikoa. 2016ko lagin-aldia. Errenteriako Udala.

Gosá, A., Bergerandi, A. 1994. Atlas de distribución de los anfibios y reptiles de Navarra. Munibe, Cienc. nat. 46: 109-189.

Gosá, A., Garin-Barrio, I. 2016. Inventario herpetológico en el término municipal de Zigoitia (Álava). Memoria final (2013-2016). Ayuntamiento de Zigoitia-Gobierno Vasco.

Kati, V., Foufopoulos, J., Ioannidis, Y., Papaioannou, H., Poirazidis, K., Lebrun, P. 2007. Diversity, ecological structure and conservation of herpetofauna in a Mediterranean area (Dadia National Park, Greece). Amphibia-Reptilia, 28(4): 517-529.

Lambert, A. 2003. Valoración económica de los humedales: un componente importante de las estrategias de gestión de los humedales a nivel de las cuencas fluviales. Convención de Humedales Ramsar.

Olano, I., Ruiz de Azua, N., Fernández, J.M., Arrayago, M.J., Bea, A. 2007. Estado de conservación de poblaciones periféricas de anfibios: tritón pirenaico Euproctus asper y sapo de espuelas Pelobates cultripes en la Comunidad Autónoma del País Vasco. Munibe, Suplemento 25: 66-73.

Pineda, E., Moreno, C.E., Escobar, F., Halffter, G. 2005. Frog, bat and dung beetle diversity in the cloud forest and coffee agroecosystems of Veracruz, México. Biol. Conserv. 19: 400410 .

SIARE. 2018. Distribución de Natrix astreptophora en el País Vasco. Disponible en: http://www.siare.herpetologica.es/. [Consulta: 8 junio 2016].

Vite-Silva, V.D., Ramírez-Bautista, A., Hernández-Salinas, U. 2010. Diversidad de anfibios y reptiles de la Reserva de la Biosfera Barranca de Metztitlán, Hidalgo, México. Rev. Mexicana Biodiver. 81 (2): 473-485. 